\title{
An IETM application research of equipment testing fault auxiliary diagnosis based on Web
}

\author{
Peng Jun ${ }^{\mathrm{a}}$, Li Hui ${ }^{\mathrm{b}}$, Wang Qingjiang ${ }^{\mathrm{c}}$, Cheng $\mathrm{Pei}^{\mathrm{d}}{ }^{\text {,Shan }} \mathrm{Xin}^{\mathrm{e}}$ \\ Naval Aeronautical and Astronautical University, Yantai, China, 264001 \\ apj00198@163.com, bkingsololi@sina.com, 'cjw16773@sohu.com, \\ dchengp1021@qq.com,newgoldth3@sina.com
}

Keywords: Fault diagnosis; IETM; Expert system

\begin{abstract}
In view of the shortcomings existing in traditional paper technical data in equipment testing and failure analysis, the article puts forward the equipment test failure aided diagnosis of IETM design method based on Web, make high complexity fault diagnosis work become easy, visualized flow process, thus improves weapons and equipment maintenance support capability.
\end{abstract}

\section{Introduction}

At present, there are various technical manuals supporting domestic complex equipment and paper is given priority to, so there are many problems existing in operation and maintenance: large volume and weight, high printing cost, long cycle, hard to update and use, etc. Various manuals are independent of each other, so assurance personnel are difficult to make failure analysis in equipment testing when found fault phenomena with comprehensive use of these technical manuals. Troubleshooting staff would use "intuition" or "thumb" in failure analysis, and excessively rely on experience and level which are likely to cause failure, false negative, cause quality hidden trouble and delay guarantee progress, or even introduce new fault or cause more serious consequences.

In order to solve these problems, develop a set of equipment test failure auxiliary diagnosis of IETM based on Web, and integrate equipment circuit signal diagram, failure phenomenon, failure analysis and elimination method or maintenance advice and other technical information in the database. This can be convenient to browse, query and update. Test equipment fault diagnosis technology manual digitization and standardization implementation provides the interactive and visualization tools for troops equipment maintenance and repair and technical training, improves work efficiency, enhances security and confidentiality, reduces total life cycle cost of equipment, and is of great significance in improving the security of troops equipment and combat ability.

\section{IETM Technology Advantages in Equipment Maintenance Support}

IETM is an interactive electronic technical manual appeared in the 1990s, and is used for complex weapon system or military and commercial equipment diagnosis and maintenance information packets. It integrates application of technologies such as computer, network, database, graphics, information processing and decrypt to organically organize and manage multifarious manuals, maintenance manuals and other information, accurately display the information needed by system operator or maintenance technicians on the electronic screen in form of optimization, and provide access to inquisition in the form of interaction to speed up equipment use and the implementation of security activities [1].

IETM is different from ordinary electronic products, and its advantage is embodied in: follow unified standard user interface, display with window optimization, display style is "graphic box wizard" rather than "page guide"; strong interactivity, the user interacts with IETM by navigation, retrieval, and other ways, get maintenance process wizard, technical data navigation, security resources supply information related to equipment maintenance support help, realize auxiliary maintenance support; Information technology is not dependent on a specific platform, and can realize 
mutual exchange of unconstrained; Have strong ability of network communication and data exchange which ensures its real-time and maintainability data content [2].

\section{Test Failure Auxiliary Diagnosis of IETM Information Data Collection and Processing}

IETM technology information has two sources: one is equipment technical paper files; the other is edited digital technology. These technical data may be used as raw material production of IETM. Dividing the data into basic information elements, DTD (Document Type Definition) define, including elements, elements in sequence, attributes and entities, etc. After defined DTD, use a DTD defined element name and attribute as a marker, mark a variety of formats of electronic documents, convert them to XML (Extensible Markup Language) documents. XML document design flow chart is shown in Figure 1.

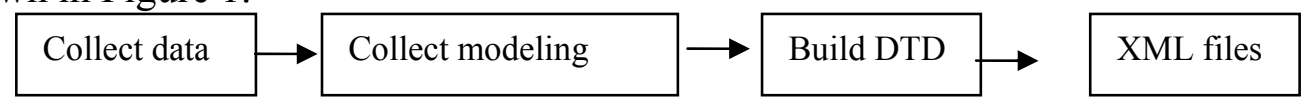

Figure 1 XML document design flow chart

\section{Test Failure Auxiliary Diagnosis of IETM Database Establishment}

Test failure auxiliary diagnosis of IETM adopts hierarchical structured design of interactive electronic manual. Class4 IETM database must use Logistic Support Analysis (LSA) method and format design, which requires "special design without redundancy relational database or object-oriented hierarchical database" [3].

When produce IETM, first define database structure, project managers make technical information classification for data editing staff in technical document analysis. According to the defined volume label set (Tag set) in data norms, specify a volume label for the analysis of documents, and deposit in database backup. Multimedia production staff makes multimedia display parts in technical information, assurance planners integrate IETM, security analysis records and other documents together, and programmers make IETM to provide comprehensive, intuitive technology information for assurance personnel.

\section{Test Failure Auxiliary Diagnosis of IETM Application Development}

In IETM development, refer to AECMAS1000D, MIL-PRF-87268A and MIL-PRF-87269A and other international standards as a reference, achieve CLASS4 function, and meet the requirements of our code for Design of Interactive Electronic Technical Manual Equipment.

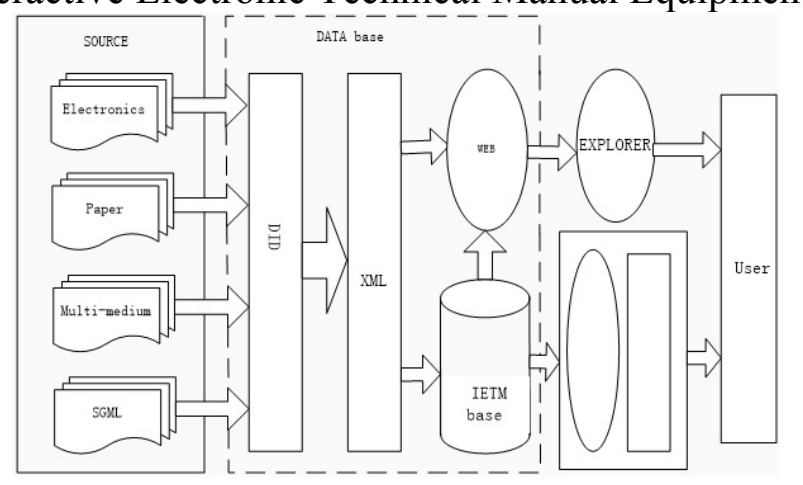

Figure 2 Test equipment fault auxiliary diagnosis of IETM development process

Equipment test failure auxiliary diagnosis of IETM development is basically divided into three stages: technical information collection, processing, and providing. Data collected can be a variety of formats, which needs a uniform format. IETM is designed according to the fourth electronic brochure: unconstrained exchange of information, need to make technical information down into basic elements, international standardized XML format, and use hierarchical structured design documentation standards. Test failure auxiliary diagnosis of IETM development process is shown in Figure 2. 
A. Three layer architecture design based on Web

IETM application framework is based on Web three layer running mode, namely browser + Web server + database server architecture model [4]. According to the implementation of logical functions, it is divided into presentation layer, business logic layer and data access layer. Presentation layer is interface of equipment support personnel and IETM system; business logic layer is the core of the three layers of application system in realizing business rules and business logic, and completing system logic control functions; data access layer contains data storage and its interaction component services, and completes data processing function. Three layers application framework based on Web is shown in Figure 3.

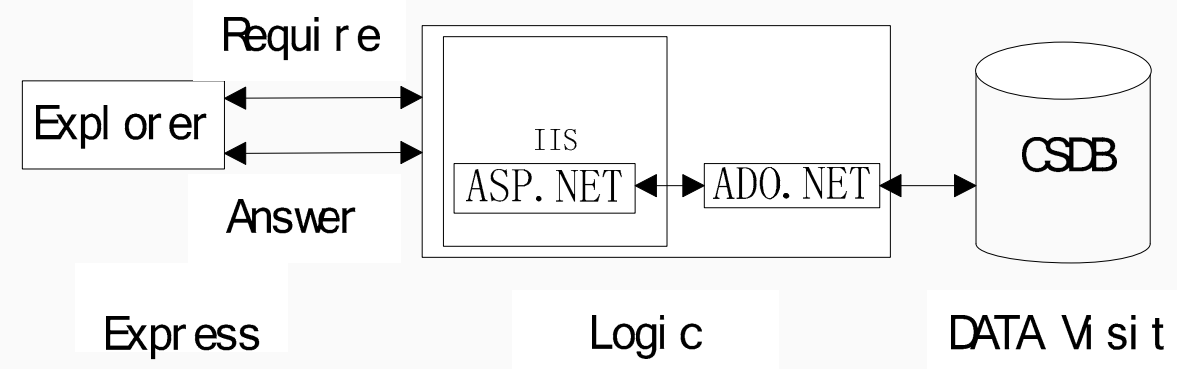

Figure 3 Three layers application framework based on Web

B. IETM browsing program design

Program adopts object-oriented c\# language and three layers frame structure development pattern, and is divided into presentation layer, logic layer, and data layer to realize network application of RIA (Rich Interface Applications) architecture which implements in Flash, Asp.net, and SQL2005 languages respectively. Flash produces front page; Asp.net reads database content, and generates a dynamic XML file; Flash reads XML file, and the user can see the content database background. Use modern Web world popular three-tier architecture theory, and face object-oriented programming. System modules structure is shown in the figure below, which includes expert knowledge fault diagnosis, experience, knowledge fault diagnosis, historical data statistics, and system settings module.

According to actual situation, considering future equipment technical support training construction needs, this paper provides a comprehensive fault diagnosis scheme for complex equipment including pilot unit, electrical unit, timer unit, radar units, comprehensive test and prior inspection. At the same time, considering the convenience of additional fault data, on the basis of expert knowledge fault diagnosis, technicians add experience fault knowledge in equipment maintenance process.

On the basis of system design and design thought, the whole system is divided into expert knowledge fault diagnosis, experience knowledge fault diagnosis, fault phenomenon query, history data statistics and system setup. It has landing, retrieving, navigation, list, data management, and other functions, achieves human-computer interaction requirements, and realizes IETM equipment testing auxiliary diagnosis application.

\section{Conclusion}

IETM is an important measure implementing United States and other developed countries modernization of equipment support. With the development of computer and network technology, establishing web-based equipment maintenance support of IETM has become the inevitable trend of development. In this paper, assisted by test equipment fault diagnosis, three layer running mode architecture model based on Web, namely browser + Web server + database server, is adopted to test failure auxiliary diagnosis application of IETM. It has a certain practical significance in improving equipment maintenance support capability. 


\section{References}

[1] Li Zongliang, Jiang Lili, Gu Zonglei. Standard IETM production research based on ASD S $1000 \mathrm{~d}$ [J]. Journal of Engineering Science and Technology, 2007.7 (19) : 4939-4943.

[2] Wang Weiguo, Zhu Mi. Air-to-air missile interactive electronic technical manual study [J]. Journal of Defense Technology Foundation. 2007.8 (8) 46-48.

[3] Song Jianshe, Cao Xiaoping, Cao Yaoqin, He Zhide. Equipment maintenance information engineering [M]. National Defense Industry Press. 2005.2:137-139.

[4] Guo Jiansheng, Liu Xuefeng. Interactive electronic technical manual research based on Web calculation model [J]. Computer Engineering. 2004.30 (4) : 145-147.

[5] Guo Li.Evaluation on effectiveness of missile maintenance information.Surface Force[J]. Fire Command and Control, 2011,7:61-64. 